\title{
CUIDADORAS INFORMAIS DE PORTUGAL: VIVÊNCIAS DO CUIDAR DE IDOSOS
}

INFORMAL CAREGIVERS IN PORTUGAL: EXPERIENCES OF CARING FOR ELDERLY

\author{
Maria João Fernandes Marques ${ }^{1}$ \\ Helena Jorge Cardoso Teixeira ${ }^{2}$ \\ Dayse Cristine Dantas Brito Neri de Souza ${ }^{3}$
}

Resumo O cuidador informal surge da necessidade de se cuidar dos doentes e idosos no domicílio, com a função de auxiliar nas medicações, higiene e alimentação sem remuneração. Esse tipo de auxílio suscita sobrecarga física, psicológica e financeira. No âmbito da qualidade do cuidar, salienta-se a necessidade de se olhar e ajudar os cuidadores informais no árduo apoio aos seus doentes, que, na maioria, são idosos. $\mathrm{O}$ objetivo deste artigo é apresentar as percepções vivenciadas pelas cuidadoras informais, bem como o reconhecimento, por elas próprias, das sobrecargas que as afetam holisticamente - emocionais, físicas, financeiras -, e a importância de desenvolver estratégias de coping na formação sobre como melhor cuidar. As entrevistadas residem nas regiões Norte e Centro de Portugal e responderam ao inquérito adaptado do The Zarit Burden Interview. As entrevistas foram analisadas com o apoio do software NVivo 8. Os dados obtidos indicam que as cuidadoras informais enfrentam mais sobrecargas físicas e emocionais do que financeiras. No entanto, salientaram que, apesar das dificuldades, gostam de cuidar dos seus familiares. Quanto a serem convidadas para participarem de formações sobre como melhor cuidar, algumas confirmaram o interesse. Verifica-se que as cuidadoras informais são carentes de uma formação que as capacite a melhor cuidar prevenindo consequências danosas.

Palavras-chave cuidadoras informais; idosos; dependentes; domicílio; vivências.
Abstract The informal caregiver arises from the need to care for sick and elderly in their homes with the task of assisting with medications, hygiene and food without payment. This kind of aid raises overhead in physical, psychological and financial. From the quality of care emphasizes the need to look at and assist caregivers in helping hard their patients, which most are elderly. The aim is to present the perceptions experienced by informal caregivers, as well as recognition of the same burdens that affect them holistically: emotional, physical, financial and the importance of developing coping strategies through training on how to better care. The interviewed in the research belong to the North and Central Portugal, answer to the survey adapted from The Zarit Burden Interview. The interviews were analyzed with the support of NVivo 8 software. The data indicate that informal caregivers face more types of physical and emotional than financial burden. However, they stressed that despite the difficulties like taking care of their families. About being invited to participate in training on how to best care, some have confirmed their interest. We found that informal caregivers are lacking of an education that enables them to better care for preventing unsafe consequences.

Keywords informal caregivers; elderly; dependents; home; experiences. 


\section{Introdução}

As alterações demográficas do último século, que se traduziram na modificação e, por vezes, na inversão das pirâmides etárias, refletindo o envelhecimento da população, vieram colocar aos governos, às famílias e à sociedade em geral desafios para os quais não estavam preparados. O prolongamento da vida associado aos avanços na medicina e a diminuição da fecundidade têm conduzido ao envelhecimento da população (Direcção Geral de Saúde, 2004). A Organização das Nações Unidas (2007) estima que o mundo terá mais de dois bilhões de pessoas com mais de 60 anos em 2050, e 80\% delas estarão nos países em desenvolvimento. Dos dois bilhões de pessoas com mais de 60 anos esperados até a metade do século, cerca de 400 milhões terão mais de 80 anos, representando um explosivo crescimento em relação aos menos de 90 milhões que existiam há dois anos.

Este aumento da longevidade ao qual Portugal não se encontra alheio, apesar de estar aquém dos padrões de alguns países europeus, causa impacto profundo na saúde pública. Neste país, as alterações na estrutura demográfica estão bem patentes quando se comparam as pirâmides de idades de 1960 e 2000. Entre 1960 e 2000, a proporção de jovens (0-14 anos) diminuiu de cerca de $37 \%$ para $30 \%$. Deve-se referir ainda que o ritmo de crescimento da população idosa é quatro vezes superior ao da população jovem (Oliveira, Rosa et al., 2008).

$\mathrm{O}$ aumento da longevidade traz consigo maior prevalência das doenças crônicas e de dependências, o que leva a pessoa doente a necessitar de ajuda parcial ou total no desempenho das suas Actividades da Vida Diária (AVDs) (Sousa, Figueiredo et al., 2004).

No entanto, os governos ficam impossibilitados de, financeiramente, responderem às necessidades da população. O cuidador informal (CI) surge, então, da necessidade de se cuidar da pessoa/familiar doente no domicílio. Segundo Pérez, Abanto e Labarta (1996), o cuidador informal é definido como a pessoa encarregada de cuidar das necessidades básicas e instrumentos da vida diária do indivíduo dependente durante a maior parte do dia, sem receber qualquer contribuição econômica por isso. Distingue-se, portanto, do prestador de cuidados formal, que é o profissional que optou voluntariamente por receber preparação acadêmica, sendo remunerado pelos cuidados que presta (Machado, 2007).

Para Duarte (1996), independentemente de o cuidador estar inserido na rede formal ou informal de prestação de cuidados, ele é um profissional que convive diariamente com a pessoa doente, auxiliando nos cuidados de higiene, alimentação, gestão do regime terapêutico e atividades reabilitadoras, interagindo com os outros profissionais no cuidar. 
Autores como Paulo et al. (2008) classificam o cuidador em primário ou secundário. O primário "é o responsável pelo cuidado diário do idoso, sendo a única pessoa a desempenhar esta tarefa. O cuidador secundário realiza a actividade ocasionalmente, de modo restrito" (Paulo et al., 2008, p. 29).

Os membros da família são os principais cuidadores informais, geralmente filhas ou esposa, predominando o sexo feminino na escolha (Grunfeld, Glossop et al., 1997; Krasch, 2003). Esse compromisso surge por obrigação ou dever, disponibilidade de tempo ou mesmo por solidariedade. Caldas (2002) salienta que a influência histórica, os imperativos culturais e os preceitos religiosos também influenciam a família a cuidar dos seus idosos. Em outras situações, como acrescentam Fernandes et al. (2002), os cuidadores assumem esse papel pelas circunstâncias e não por escolha, mesmo tendo o reconhecimento que essa deva ser a sua nova missão.

Um número considerável de cuidadores informais, por carecerem de uma formação adequada, tem maior risco de sobrecarga física, emocional, social e financeira. Muitas das vezes, o cuidador informal desempenha o seu papel sozinho, sem qualquer ajuda; isso leva a que experimente cansaço, estresse, exaustão, mas também bem-estar, afeição e ternura por cuidar de alguém de quem gosta. Cuidar de um idoso, dependente, portador de uma doença crônica, pode representar uma ameaça constante para o cuidador informal se ele não reunir estratégias de coping 4 eficazes para lidar com a situação (Zarit, 1997).

Diversos estudos relatam as consequências que os cuidadores informais e os familiares enfrentam ao cuidarem de seus doentes no domicílio. Como dizem Martins, Ribeiro e Garrett (2003), “a doença, qualquer doença, é sempre uma situação de crise, um acontecimento stressor, que produz efeitos no doente e na família".

Pelo que foi anteriormente descrito, os cuidadores informais, por carecerem de uma formação que os prepare para cuidar, podem experimentar sobrecarga e por isso necessitam de estratégias que os auxiliem. Segundo Braithwaite (1992), a sobrecarga que o cuidador informal experimenta advém da falta de conhecimento no cuidar e da incapacidade mental do doente, que torna o papel irreversível e de completa dependência. A doença prolongada torna a situação geradora de estresse, o que afeta o equilíbrio normal do funcionamento familiar nos aspectos pessoal e social (Rabow, Hauser et al., 2004).

Nunes (1995) refere que a formação é útil na preparação para situações novas ou inesperadas da vida, dado que permite a "aquisição de conhecimentos e de valores, desenvolvimento de capacidades e atitudes, mudança de representações, preparação para intervenção social, modificação de perfis sociais e profissionais e desenvolvimento de identidades" (Nunes, 1995, p. 238). 
Assim, a formação dos cuidadores poderá promover um processo de reflexão sobre a experiência do cuidar, apreender novas formas e técnicas, maior socialização entre o profissional de saúde e o doente e, por fim, um reconstruir de intenção e vontade de produzir efeitos em si e nos indivíduos. Isso repercute positivamente na garantia da integridade física e emocional dos cuidadores (Padilla, 2008).

O objetivo primário deste trabalho foi apresentar as percepções e situações vivenciadas pelas cuidadoras informais acerca do cuidar do familiar idoso dependente, ao mesmo tempo que reconhecer situações geradoras de sobrecarga passíveis de serem enfrentadas por meio de formação adequada.

\section{Método}

O trabalho apresentado 5 é de natureza qualitativa numa abordagem descritiva, cujo objetivo passa por descrever características de determinada população ou fenômeno (Gil, 1995; Steubert, 2002). Assim, pretende-se que, por meio das falas das cuidadoras, se consiga uma análise mais concreta das percepções dos cuidadores informais: como se sentem e como vivenciam essa experiência.

A seleção das participantes foi por conveniência, tendo como critério único serem cuidadoras de idosos dependentes no domicílio e de localidades diferentes de Portugal (Norte e Centro). Os contatos foram estabelecidos em parceria com enfermeiros das Unidades de Saúde Familiar e contatos pessoais. Foram entrevistadas duas cuidadores informais de Braga, duas da Trofa, duas de Ovar e duas de Aveiro, que voluntariamente aceitaram participar do estudo, num total de oito participantes e seis horas de duração.

Os itens analisados foram a idade, o sexo, o estado civil, a profissão, as habilitações acadêmicas, o parentesco e a doença da pessoa dependente/doente. A recolha de dados foi realizada de abril a junho de 2010. Definiu-se, dentre os instrumentos analisados, o roteiro de entrevista de The Zarit Burden Interview, o qual correspondeu aos objetivos deste estudo. Na escala original, apresenta-se na forma de 22 perguntas que avaliam o impacto de doenças mentais e físicas nos cuidadores informais. $\mathrm{O}$ escore varia de 0 a 88 - quanto maior a pontuação, maior a sobrecarga. Esse instrumento já foi validado para a língua portuguesa por Scazufca (2002)6 e Sequeira (2010). ${ }^{7}$ No entanto, para responder aos objetivos do estudo e para melhor contextualização da realidade das cuidadoras informais, utilizou-se o instrumento adaptado e validado para a população portuguesa, com perguntas abertas, permitindo assim respostas mais amplas, com a possibilidade de se trabalhar melhor o conteúdo e de se obterem dados mais ricos. O roteiro foi constituído por oito temas e 35 questões, desde os dados pessoais aos tipos de 
sobrecarga, reações às exigências do cuidar, apoio externo e formação. As entrevistas foram gravadas após autorização verbal por parte das cuidadoras e posteriormente transcritas.

A análise dos dados foi feita com o apoio do software NVivo8 (Lage, 2011), da empresa QSR International. Definiu-se como categorias principais: apoio para cuidar, apoio familiar, capacidade para cuidar, sobrecarga emocional, física, financeira, social e a importância da existência de uma formação para melhor cuidar. Após realizados todos os cruzamentos por meio de matrizes, obtiveram-se os resultados.

Os entrevistados foram todos do sexo feminino, duas solteiras, uma viúva e cinco casadas, com idades compreendidas entre os 30 e os 71 anos. O nível de escolaridade varia entre o $1^{\circ}$ ciclo (corresponde ao ensino fundamental) e o ensino superior, sendo que cinco delas são donas de casa, uma está em licença de trabalho, uma é advogada e uma é assistente operacional. As entrevistas foram realizadas nos Centros de Saúde das localidades e nas moradias e tiveram entre uma e duas horas de duração. Quanto ao grau de parentesco dos familiares dependentes, identificaram-se mães, pai, sogro, marido e avó.

Com a finalidade de analisar o estado emocional das cuidadoras informais de idosos e o tipo de doença do familiar dependente, as categorias que emergiram das respostas obtidas nas entrevistas foram, nomeadamente: cansaço emocional; compromisso com o cuidar; gosto pelo cuidar; isolamento; perturbação mental; planos para o futuro; privacidade; e sentimento de culpa. No Quadro 1, apresenta-se um panorama geral dessa dimensão.

\begin{tabular}{lcccc} 
Quadro 1 & \multicolumn{3}{l}{} \\
\hline Estado emocional do cuidador informal e tipo de doença do familiar dependente & Alzheimer & Paraplegia & Total \\
\hline & 11 & 5 & 4 & 20 \\
\hline Cansaço emocional & 3 & 0 & 2 & 5 \\
Compromisso de cuidar & 16 & 8 & 4 & 28 \\
Gosto de cuidar & 4 & 3 & 1 & 8 \\
Isolamento & 0 & 0 & 0 & 0 \\
Perturbação mental & 0 & 0 & 0 & 0 \\
Planos para o futuro & 0 & 0 & 0 & 0 \\
Privacidade & 0 & 0 & 0 & 0 \\
Sentimento de culpa & 34 & 16 & 11 & 61 \\
Total & & & &
\end{tabular}

Fonte: As autoras. 
Relativamente à frequência de unidades de registro, os resultados indicam que as cuidadoras informais que cuidam de familiares idosos com acidente vascular cerebral (AVC) apresentam números acentuados $(\mathrm{n}=34)$ de incidências, comparativamente a familiares com outras patologias. Tal situação justifica-se pelo fato de doenças cerebrovasculares causarem sequelas incapacitantes, limitando os pacientes ao nível motor, sensitivo, sensorial e cognitivo, alterando a dinâmica de vida dos doentes, que necessitam de apoio e acompanhamento diário (Perlini e Faro, 2005), sobrecarregando as cuidadoras informais na prestação dos cuidados.

É de se salientar que o processo de cuidar dos familiares dependentes, apesar de as cuidadoras informais declararem que gostam de cuidar $(n=16)$, alterou a sua vida pessoal, levando à falta de privacidade e causando isolamento social.

(...) ando com os nervos às vezes... tristeza... à uma porque estes anos têm sido muito diferentes, e depois estar metida em casa (Ovar 1).

Só quando às vezes passa horas e horas, dias e dias sem dormir. Chega a um ponto que já não durmo nada e fico a pensar: ai, meu Deus, que não consigo... (Ovar 2).

Outra dimensão de análise, alvo de interesse, foi relativamente às condições físicas das cuidadoras informais comparativamente à idade. O Quadro 2 indica os resultados obtidos por unidades de registro.

Quadro 2

\begin{tabular}{lccc}
\hline Idade e condição física das cuidadoras informais & & \\
\hline Idade & Cansaço físico & Doença física & Total \\
\hline 30 a 35 anos & 3 & 0 & 3 \\
36 a 41 anos & 1 & 0 & 1 \\
42 a 47 anos & 0 & 0 & 0 \\
48 a 53 anos & 2 & 1 & 3 \\
54 a 59 anos & 4 & 1 & 5 \\
60 a 65 anos & 1 & 0 & 1 \\
66 a 71 anos & 4 & 1 & 5 \\
Total & 15 & 3 & 18 \\
\hline
\end{tabular}

Fonte: As autoras. 
Numa avaliação global do estado físico das cuidadoras informais, o Quadro 2 revela que houve maior número de unidades de registro $(n=14)$ com maior incidência nas faixas etárias entre 54 e 59 anos e 66 e 71 anos, que apresentaram repercussões físicas, distinguindo-se cansaço físico geral, dores nas costas e pernas e consequente agravamento nas condições musculares e ortopédicas.

Bocchi (2004) destaca que essa situação leva muitos cuidadores informais a não terem tempo para as suas próprias necessidades, devido ao pouco tempo para si mesmos.

(...) por esse sentar, levantar, estou sentindo dor nas costas... ah, pois que dói, não é? Vai doendo, e a gente fica cansada... mas a gente vai dando um jeito (Aveiro 2).

Agora até ando a fazer muitos exames, agora até, de hoje a oito dias, não, na segundafeira vou fazer uma prova de esforço, tive a fazer uns exames aos pulmões, porque no outro dia apanhei aqui um esforço aqui na cabeça, e tenho andando assim também muito... também tenho o colesterol que tem tendências a subir, mas isto é próprio agora da idade (Aveirol).

A análise do Quadro 3 faz surgir como evidência a relação entre o tipo de parentesco familiar e as despesas financeiras no cuidar do familiar idoso dependente.

Quadro 3

\begin{tabular}{|c|c|c|c|c|c|c|c|}
\hline Parentesco familiar e despesas fina & ras no $\mathrm{C}$ & & & & & & \\
\hline & Mãe & Pai & Marido & Sogro & Mãe e Pai & Avó & Total \\
\hline Ajuda segurança social & 1 & 0 & 2 & 0 & 0 & 0 & 3 \\
\hline Enfermagem setor público & 4 & 1 & 0 & 1 & 1 & 1 & 8 \\
\hline Pagamento serviços domiciliários & 0 & 0 & 0 & 1 & 0 & 0 & 1 \\
\hline Sem apoio institucional & 0 & 0 & 1 & 0 & 0 & 0 & 1 \\
\hline Familiar & 1 & 0 & 1 & 3 & 0 & 0 & 5 \\
\hline Despesas pessoais com o doente & 1 & 1 & 0 & 2 & 0 & 0 & 4 \\
\hline Total & 7 & 2 & 4 & 7 & 1 & 1 & 22 \\
\hline
\end{tabular}

Fonte: As autoras. 
Verifica-se que houve mais unidades de registros das cuidadoras informais que cuidam da mãe e do sogro $(n=7)$, bem como uma unidade de registro em que a cuidadora não aceitou apoio domiciliar de enfermagem por considerar que não precisava. Duas unidades de registro destacam o pedido de subsídio da Segurança Social (instituição do governo português que presta apoio social e financeiro) para o cuidar do seu familiar dependente, enquanto outra cuidadora solicitou apoio da Santa Casa de Misericórdia (instituição particular de solidariedade social, ligada à Igreja Católica) duas vezes por semana. A cuidadora informal que salientou essa necessidade afirmou que precisaria de mais dias; no entanto, as condições financeiras impediam de obter mais apoio domiciliar.

Por vezes, o fato de o cuidador informal não solicitar o apoio domiciliar com mais frequência deve-se à falta de colaboração financeira por parte da família (Andrade, 2009).

Segundo Lacerda e Valla (2003), o apoio social contribui para o enfrentamento dos problemas de saúde-doença, na medida em que ajuda os sujeitos a terem maior controle das situações estressantes. Segundo Bocchi (2004), as mudanças financeiras na família podem contribuir para as limitações encontradas na vida social dos cuidadores.

Não preciso de ajuda de ninguém, consigo fazer tudo sozinha (Aveiro 2).

E apoio para o colchão tive de ir pedir à Santa Casa, estava a pagar sete euros e cinquenta cêntimos só daquele colchão de cima e em princípio tinha a cama articulada, pagava trinta e qualquer coisa em euros (Trofa 2).

Outro aspecto de nota é a ênfase na relação entre a profissão das cuidadoras informais de idosos e a intenção em participar de uma formação em como melhor cuidar. Nesse sentido, o Quadro 4 aponta para alguns resultados.

Quadro 4

Profissão das cuidadoras informais e formação

\begin{tabular}{lccccc}
\hline & $\begin{array}{c}\text { Aceita } \\
\text { formação }\end{array}$ & $\begin{array}{c}\text { Impossibilidade } \\
\text { de se ausentar }\end{array}$ & $\begin{array}{c}\text { Impossibilidade } \\
\text { de tempo }\end{array}$ & $\begin{array}{c}\text { Não precisa } \\
\text { de formação }\end{array}$ & Total \\
\hline Doméstica & 5 & 0 & 0 & 0 & 5 \\
Empregada de balcão & 0 & 1 & 0 & 0 & 0 \\
Operária fabril & 2 & 0 & 0 & 1 & 2 \\
Feirante & 0 & 0 & 2 & 3 \\
\hline
\end{tabular}


Continuação - Quadro 4

\begin{tabular}{lccccc}
\hline Profissão das cuidadoras informais e formação & $\begin{array}{c}\text { Aceita } \\
\text { formação }\end{array}$ & $\begin{array}{c}\text { Impossibilidade } \\
\text { de se ausentar }\end{array}$ & $\begin{array}{c}\text { Impossibilidade } \\
\text { de tempo }\end{array}$ & $\begin{array}{c}\text { Não precisa } \\
\text { de formação }\end{array}$ & Total \\
\hline Advogada & 1 & 0 & 0 & 0 & 1 \\
Assistente operacional & 1 & 0 & 0 & 0 & 1 \\
Total & 9 & 1 & 2 & 1 & 13
\end{tabular}

Fonte: As autoras.

Em relação ao Quadro 4, constata-se que houve nove unidades de registro das cuidadoras informais respondentes que revelaram interesse em realizar formação em como melhor cuidar dos seus familiares dependentes. No entanto, foi afirmado uma vez que não havia necessidade de formação. Outra unidade de registro revelou a impossibilidade de participar em alguma formação por não ter tempo.

Estudos internacionais reforçam a necessidade de os cuidadores informais receberem formação em como melhor cuidar dos seus familiares dependentes, tendo em conta as tarefas realizadas serem inadequadas e provocarem acentuados problemas físicos, emocionais, psicológicos e pessoais (Andrade, 2009; Kurz, Wagenpfeil et al., 2010).

Formações já desenvolvidas revelaram significativos sucessos na formação dos cuidadores informais no âmbito de maior partilha e interação com outros cuidadores acerca das suas inquietudes, bem como novos conhecimentos sobre as doenças, sugestões em como melhor cuidar, mudança nas ideias preconcebidas e consciencialização do grupo de enfermagem em oferecer habilidades básicas no acompanhamento dos familiares e pacientes dependentes (Mahoney, 1994; Padilla, 2008; Gelmini, Morabito et al., 2009).

O reforço em programas de intervenção na área formativa, de modo a capacitar o cuidador informal, promove um aumento de competências, diminuindo-lhe a sobrecarga, e um aumento da sua qualidade de vida e da própria pessoa idosa a quem presta cuidados (Júnior, Corrente et al., 2011).

Acho que era importante... há tanta gente que tem muitas dúvidas, que não tem apoios, que se sente sozinha... cansada... Também temos de olhar por nós, por quem dedica a sua vida a cuidar de quem precisa, não é? E se houvesse a possibilidade de eu participar, sim, iria (Braga 2). 
Não, não preciso, por acaso não preciso, tenho todo o jeito, mesmo para o deitar e tudo, também tenho os meus filhos, tá a ver a gente (Aveiro 1).

Com este estudo, foi possível verificar que a realidade das cuidadoras informais das localidades das regiões Norte e Centro de Portugal confirma as problemáticas descritas na literatura internacional relativamente aos efeitos da sobrecarga e ao fato de os cuidadores informais serem predominantemente do sexo feminino. Os resultados revelam que as cuidadoras informais sofrem sobrecargas objetivas e subjetivas com alterações nas suas vidas pessoais, sociais, profissionais, físicas e emocionais.

Constata-se também que o instrumento The Zarit Burden Interview adaptado correspondeu aos objetivos da investigação. As cuidadoras informais sentiram-se à vontade e relataram com transparência as respostas.

Confirma-se que todas as entrevistadas apresentaram desgaste emocional e problemas psicopatológicos devido ao fato de cuidarem sozinhas do familiar e em face do tipo e da gravidade da(s) doença(s). Os principais sintomas são a limitação no tipo de cuidar, isolamento, cansaço emocional e físico.

Algumas cuidadoras familiares rejeitaram ajuda de outros familiares por considerarem ser sua obrigação fazê-lo exclusivamente. Uma cuidadora informal declarou não precisar de apoio da assistência domiciliária. No entanto, conclui-se que as entrevistadas revelaram sobrecarga física ao cuidar do seu familiar dependente, causando fadiga crônica.

Acerca da sobrecarga financeira, destaca-se uma unidade de registro na qual foram solicitados os serviços de apoio da Santa Casa de Misericórdia.

As cuidadoras que tinham ocupação profissional foram forçadas a abandoná-la para cuidar do familiar dependente e, dessa forma, não têm planos para o futuro enquanto estiverem a exercer a prestação de cuidados.

Relativamente à questão de participarem em alguma formação em como melhor cuidar do seu familiar dependente, destacaram interesse em participar na formação; no entanto, apresentaram dificuldades pela falta de tempo e a impossibilidade de se ausentarem do domicílio.

\section{Conclusão}

O estudo desenvolvido destaca o impacto de um idoso dependente e sua doença na vida do familiar que assume a responsabilidade principal pelo cuidado domiciliar. Com base nos depoimentos das oito cuidadoras informais entrevistadas, pôde-se compreender a extensão das dificuldades e implicações negativas que o cuidar pode impor aos cuidadores quando não apoiados e orientados. O despreparo do cuidador pode trazer sérios prejuízos quer ao próprio quer à pessoa doente, resultando, inclusive, em frequentes 
hospitalizações. Além disso, o despreparo pode gerar ansiedade e maior desgaste físico, ocasionando situações de risco para ambos.

Os dados obtidos neste estudo e a aproximação do conhecimento já produzido sobre o tema apontam para a necessidade de programas sociais de assistência às famílias que apresentam um integrante dependente para a realização das atividades quotidianas.

\section{Colaboradores}

Maria João Fernandes Marques, Helena Jorge Cardoso Teixeira e Dayse Cristine Dantas Brito Neri de Souza participaram de todas as etapas de preparação do manuscrito, que consistiram na colheita de dados, redação e revisão do texto.

\section{Notas}

1 Graduanda de Licenciatura em Enfermagem da Escola Superior de Saúde da Universidade de Aveiro, Aveiro, Portugal.<mariamarques@ua.pt>

Correspondência: Universidade de Aveiro, Campus de Santiago, CEP 3810-193, Aveiro, Portugal.

2 Técnica de Investigação da Universidade de Aveiro, Aveiro, Portugal. Licenciada em Gerontologia pela Escola Superior de Saúde de Bragança, Portugal. <helena.teixeira@ua.pt>

3 Investigadora auxiliar do Departamento de Educação da Universidade de Aveiro, Aveiro, Portugal. Doutora e pós-doutora em Ciências da Educação. <dayneri @ua.pt>

4 Segundo Gomes, R. \& Pereira, A. (2008), os mecanismos de coping são estratégias para lidar com situações difíceis.

5 Este estudo foi realizado em tempo parcial ao longo de um ano pela estudante de Licenciatura em Enfermagem Maria João Marques Fernandes, como bolsista da Fundação para a Ciência e a Tecnologia (FCT) de Iniciação à Investigação, e pela licenciada em Gerontologia Helena Jorge Cardoso Teixeira, bolsista técnica de investigação da FCT. Foi promovido pelo Centro de Investigação Didáctica e Tecnologia na Formação de Formadores (CIDTFF), estando integrado num projeto maior, financiado pela FCT, intitulado "Cuidadores informais de idosos: do levantamento das necessidades ao desenvolvimento de estratégias de intervenção", tendo como responsável Dayse Neri de Souza, investigadora auxiliar do Departamento de Educação da Universidade de Aveiro. 
6 Versão brasileira da escala The Zarit Burden Interview.

7 Versão portuguesa da escala The Zarit Burden Interview.

\section{Referências}

ANDRADE, Ana Margarida E. Escuela de Cuidadores como programa psicoeducativo para cuidadores informales de adultos mayores con demencia. Revista Cubana de Salud Pública, El vedado, Habana v. 32, n. 2, p. 1-14, 2009.

BOCCHI, Silvia Cristina M. Vivenciando a sobrecarga ao ser um cuidador familiar de pessoa com Acidente Vascular Cerebral (AVC): análise do conhecimento. Revista Latino-Americana de Enfermagem, Ribeirão Preto, v. 12, n. 1, p. 115-121, 2004.

BRAITHWAITE, Valerie. Caregiving burden, making the concept scientifically useful and policy relevant. Research on Aging, v. 14, n. 1, p. 3-27, 1992.

CALDAS, Célia Pereira. Contribuindo para a construção da rede de cuidados: trabalhando com a família do idoso portador de síndrome demencial. Textos sobre Envelhecimento, Rio de Janeiro, UERJ, v. 4, n. 8, p. 39-56, 2002.

DIRECÇÃO GERAL DE SAÚDE. Programa Nacional para a Saúde das Pessoas Idosas. Lisboa: Ministério da Saúde, 2004.

DUARTE, Maria Jalma Rodrigues Santana. Cuidadores? Por que e para quê? Atenção aos idoso no domicílio. Revista de Enfermagem, v. 34, n. extra, p. 126-130, 1996.

FERNANDES, Margarida, et al. Sobrecarga física, emocional e social nos cuidadores informais de doentes com AVC. Sinais Vitais, Coimbra, v. 43, p. 31-35, 2002.

GELMINI, Giovanni; MORABITO, Bartollo; BRAIDI, Giovanni. Educational and forma- tive training reduce stress in the caregivers of demented patients. Archives Gerontology and Geriatrics. n. 1, v. 1, p. 119-124, 2009.

GIL, António Carlos. Métodos e técnicas de pesquisa social. São Paulo: Atlas, 1995.

GOMES, Rosa. Maria Silva; PEREIRA, Anabela. Maria Sousa. Estratégias de Coping em educadores de infância portugueses Coping em educadores. Revista Semestral da Associação Brasileira de Psicologia Escolar e Educacional (ABRAPEE), v. 12, n. 2, p. 319-326, 2008.

GRUNFELD, Eva. et al. Caring for elderly people at home: the consequences to caregivers. Canadian Medical Association Journal, v. 15, p. 1101-1105, 1997.

JÚNIOR, Paulo Roberto R. et al. Efeito da capacitação dos cuidadores informais sobre a qualidade de vida de idosos com déficit de autocuidado. Ciência \& Saúde Coletiva, Rio de Janeiro, v. 16, n. 7, p. 3131-3138, 2011.

KRASCH, U. Idosos dependentes: famílias e cuidadores. Cadernos de Saúde Pública, Rio de Janeiro, v. 19, n. 3, p. 861-866, 2003.

KURZ, Alexander. et al. Evaluation of a brief educational program for dementia carers: the AENEAS Study. International Journal of Geriatric Psychiatric, Manchester, v. 25, n. 8, p. 861-869, 2010.

LACERDA, Alda; VALLA, Victor. Homeopatia e apoio social: repensando as práticas de integralidade na atenção e no cuidado à saúde. In: PINHEIRO, R.; MATTOS, R. (Eds.). 
Construção da integralidade: cotidiano, saberes e práticas em saúde. Rio de Janeiro: IMS/Uerj; Cepesc; Abrasco, 2003.

LAGE, Maria Campos. Utilização do software NVivo e m pesquisa qualitativa: uma experiência em EaD. ETD - Educação Temática Digital, Campinas, v. 12, n. n.esp, p. 198-226, 2011. ISSN 1676-2592.

MACHADO, Ana Larissa G. O fazer do cuidador familiar: significados e crenças. Revista Brasileira de Enfermagem, Brasília, v. 60 , n. 5, p. 530-534, 2007.

MAHONEY, Diane F.; SHIPPEE-RICE, Raelene. Training family caregivers of older adults: a program model for community nurses. Journal of Community Health Nursing, v. 11, n. 2, p. 71-78, 1994.

MARTINS, Teresa; RIBEIRO, José Pais.; GARRETT, Carolina. Estudo de validação do questionário de avaliação da sobrecarga para cuidadores informais. Psicologia, Saúde \& Doenças, Lisboa, v. 4, n. 1, p. 131-148, 2003.

NUNES, Luísa. As dimensões formativas dos contextos de trabalho. Inovação, Lisboa v. 8, n. 3, p. 233-249, 1995.

OLIVEIRA, Catarina Resende de; et al. Estudo do perfil da população portuguesa. Coimbra: Gabinete Editorial de Relações Públicas e Imagem da Faculdade de Medicina da Universidade de Coimbra, 2008.

ORGANIZAÇÃO DAS NAÇÕES UNIDAS. Estudo Económico e Social Mundial, 2007. 2007. Disponível em: <www.un.org/esa/ analysis/wess/>. Acesso em: 27 set. 2010.

PADILLA, Orfa Nineth Morales. Efectos del programa cuidando al cuidador, en la mejora de la habilidad de cuidado. Avances en Enfermería, Bogotá, v. 26, n. 2, 27-34, jul.dez. 2008.

PAULO, Michelle Gassen. et al. Avaliação da qualidade de vida de cuidadores de idosos portadores de deficiência auditiva: influên- cia do uso de próteses auditivas. Arquivo Internacional de Otorrinolaringologia/International Archive of Otorhinolaryngology, São Paulo, v. 12, n.1, p. 28-36, 2008.

PÉREZ, Trulen. ABANTO, Alda; LABARTA, Mancho. El síndrome del cuidador en los procesos de deterioro cognitivo (demencia). Atención Primaria, v. 18, n. 4, p. 194-202, 1996.

PERLINI, Nara Marilene O. G.; FARO, Ana Cristina M. Cuidar de pessoa incapacitada por acidente vascular cerebral no domicílio: o fazer do cuidador familiar. Revista da Escola de Enfermagem da USP, São Paulo, v. 39, n. 2, p. 154-163, 2005.

RABOW, Michael W.; HAUSER, Joshua M.; ADAMS, Jocelia. Supporting family caregivers at the end of life: "they don't know what they don't know". JAMA, San Francisco, v. 291, n. 4, p. 483-491, 2004

SCAZUFCA, Marcia. Brazilian version of the burden interview scale for the assessment of burden of care in carers of people with mental illnesses. Revista Brasileira de Psiquiatria, São Paulo, v. 24, n. 1, p. 12-17, 2002.

SEQUEIRA, Carlos Alberto da Cruz. Adaptação e validação da escala de sobrecarga do cuidador de Zarit. Referência, Coimbra, v. 2, n. 12, p. 9-16, 2010.

SOUSA, Liliana; FIGUEIREDO, Daniela; CERQUEIRA, Margarida. Envelhecer em família: os cuidados familiares na velhice. Lisboa: Âmbar, 2004.

STEUBERT, Helen J. Investigação qualitativa em enfermagem. Loures: Lusociência, 2002.

ZARIT, Steven. H. Interventions with family caregivers: a guide to psychotherapy and aging. Washington, D.C.: American Psychological Association, 1997.

Recebido em 21/07/2011

Aprovado em 22/10/2011 